\title{
Editorial
}

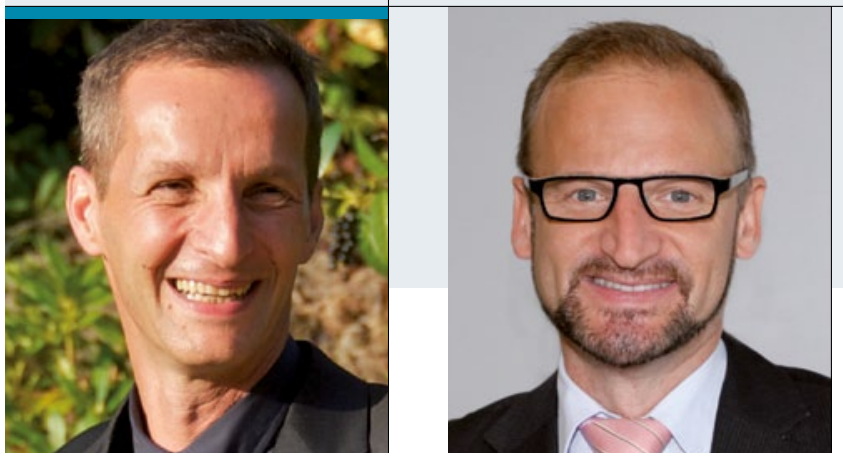

"Als Sektion HNO der DGAKI freuen wir uns besonders, dass das Allergo Journal diesem Thema besonderen Platz einräumt."

PD Dr. Martin Wagenmann, Hals-Nasen-Ohren-Klinik, Universitätsklinikum Düsseldorf

PD Dr. Oliver Pfaar, Zentrum für Rhinologie und Allergologie Wiesbaden

\section{Immer der Nase nach ... Rhinologie im Fokus}


ie allergischen Erkrankungen der oberen Atemwege sind die häufigsten Manifestationen der Atopie. Daher freuen wir uns als Sektion HNO der Deutschen Gesellschaft für Allergologie und klinische Immunologie (DGAKI) besonders, dass diesem Thema in dieser und den folgenden Ausgaben des Allergo Journal besonderer Platz eingeräumt wird. Die Übersichtsarbeiten sind nicht nur für HNO-Ärzte, sondern auch für die weiteren Fachgruppen der allergologisch tätigen Ärzte von besonderer Relevanz, da sich die Beschwerden der Patienten, die wir Allergologen täglich betreuen, nicht an die tradierten Fächergrenzen halten und eine ganzheitlich-interdisziplinäre allergologische Betreuung unerlässlich ist.

Aber nicht nur im klinischen, sondern auch im wissenschaftlichen Bereich nehmen die chronischen all-

\footnotetext{
Beitragsserie „HNO" auf einen Blick

_-Staphylokokken bei chronischer Rhinosinusitis mit Nasenpolypen. Weiß D et al. (S. 187)

_Welche Rolle spielt lgE bei chronischer Rhinosinusitis mit Polyposis nasi? Olze H et al.

_Rhinitis - allergisch oder nicht? Glowania A et al.

_Medikamentöse Therapie der allergischen Rhinitis - State of the Art 2012. Michels A et al.

_Akupunktur bei allergischer Rhinitis. Hauswald B et al.

_ Analgetika-Intoleranz. Kirsche HP et al.

_Das Surfactant-System der oberen Atemwege:

Aufbau, Funktion und klinische Bedeutung. Klimek L et al.

_Angioödeme aus Sicht der HNO. Bas M et al.

Zusätzlich in dieser Ausgabe:

_S2-Leitlinie Rhinosinusitis. Stuck B et al. (S. 165)
}

ergischen und nicht-allergischen Entzündungen der Nase, der Nasennebenhöhlen und der oberen Atemwege eine besondere Rolle ein. Die ausgesprochen gute Erreichbarkeit der betroffenen Schleimhäute eröffnet Möglichkeiten, Untersuchungen an diesen Geweben durchzuführen, die z. B. im Bereich des Bronchialsystems nur mit großem Aufwand oder gar nicht möglich sind. Gleichzeitig bestehen aber enge Zusammenhänge zwischen der chronischen Sinusitis (insbesondere mit Nasenpolypen) und dem Asthma bronchiale - sowohl was die Ätiologie als auch die Pathophysiologie dieser wichtigen Erkrankungen angeht.

Die hier vorgestellten Artikel stellen eine kleine Auswahl der verschiedenen Aspekte klinischer und wissenschaftlicher Arbeiten aus dem rhinologischen Blickfeld dar. Sie sollen daher auch keinen vollständigen Überblick der Aktivitäten der allergologisch tätigen HNO-Ärzte darstellen, sondern vielmehr dazu motivieren, weitere Arbeiten aus dem HNO-Bereich zur Publikation im Allergo Journal einzureichen. Die Zeitschrift hat eine hohe Sichtbarkeit und bietet damit die besondere Möglichkeit, auch die weiteren allergologischen Fachbereiche zur Diskussion und Kooperation anzuregen.

Zum Auftakt der Serie finden Sie in diesem Heft den Beitrag von Weiß et al. zur Rolle der Staphylokokken in der chronischen Rhinosinusitis mit Nasenpolypen (S. 187) und die aktuelle Leitlinie zur Rhinosinusitis der Deutschen Gesellschaft für Hals-Nasen-Ohrenheilkunde (S. 165). Was in den nächsten Ausgaben folgen wird, sehen Sie im Kasten links. Eine interessante Lektüre wünschen Ihnen

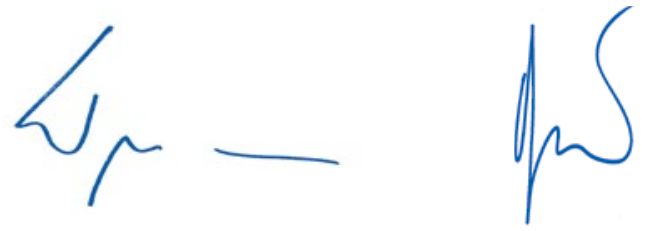

